\title{
A Social Context-Aware Recommender of Itineraries Between Relevant Points of Interest
}

\author{
Dario D'Agostino, Fabio Gasparetti, Alessandro Micarelli, \\ and Giuseppe Sansonetti $\left.{ }^{(}\right)$ \\ Department of Engineering, Roma Tre University, Via della Vasca Navale 79, \\ 00146 Rome, Italy \\ dario.dagostino@outlook.com, \{gaspare,micarel,gsansone\}@dia.uniroma3.it
}

\begin{abstract}
In this paper, we present a personalized recommender system able to suggest to the target user itineraries that both meet her preferences and needs, and are sensitive to her physical and social contexts. The recommendation process takes into account different aspects: in addition to the popularity of the points of interest (POIs), inferred by considering, for instance, the number of check-ins on social networking services such as Foursquare, it also includes the user's profile, the current context of use, and the user's network of social ties. The system, therefore, consists of four main modules that accomplish the following tasks: (1) the construction of the user's profile according to her interests and tastes; (2) the creation of the path graph in the user's proximity; (3) the routing to locate the first $k$ itineraries that match the query; (4) their ranking through a scoring function that considers the POI popularity, the user's profile, and her physical and social context. The proposed system was evaluated on a sample of 40 real users. Experimental results showed the effectiveness of the proposed recommender.
\end{abstract}

Keywords: Location-based services $\cdot$ Social networks $\cdot$ Recommender systems

\section{Introduction}

Location-based social networks (LBSNs) allow users to retrieve points of interest in the area surrounding their current location, and to share with their friends contents related to it, such as photos and geo-tagged texts. The large amount of geographical and social data made available by LBSNs offers an unprecedented opportunity to study the ways in which humans interact with their surroundings based on their social and spatial behavior [1,2]. This paper describes a recommender system of popular itineraries, which enhances the recommendation process with the user profile, the current context of use, and information extracted from social networks. For popular itineraries we mean tourist itineraries including venues deemed of interest by the user community. The user 
community is extracted from Foursquare ${ }^{1}$, a social networking service that allows users to share locations with their friends through the check-in functionality.

\section{The Recommender System}

In the proposed recommender system (RS) each user is profiled as a vector of weights whose values (between 0 and 1 ) express the user's interest in a certain category of points of interest (POIs). Such user profile is explicitly created and implicitly updated. Another crucial factor in the recommendation process is the current physical context, namely, any information that can be used to characterize the situation of an entity [3]. In this case, entities are the active user and the POIs, whilst information concerns the current location, the time of the day, the day of the week, the weather conditions, the means of transport, and so on. Almost all this information can be determined without the user's involvement. Indeed, the location is detected by the GPS sensor of the mobile device, as well as the means of transport is detected by the accelerometer. Moreover, the weather conditions are obtained from query weather services, based on the current location. Since the main goal is the recommendation of popular routes, the problem has been modeled as the search of a directed graph. Each node represents a POI (i.e., one of the Foursquare venues), each edge represents a direct link between two POIs, with a weight denoting their distance in terms of time (minutes). For the graph construction, we have to select the set of POIs, and then derive the set of edges among them. Therefore, the first step is to select the rectangular region containing those POIs, which is delimited by latitudes and longitudes of the starting point (deducted from the GPS sensor of the device), and the end point (entered by the user). After defining the region boundaries, all the POIs included in the database that fall within this area, form the graph nodes. Such POIs are then filtered based on the contextual information. For instance, time and weather conditions can be used to rule out all the POIs that would be not valid for the current situation. The edge inference and the graph construction occur as follows. The information related to an edge comprises the shortest path to get from one node to another and the traveling time, taking into account the user's means of transport. Obviously, if the user is walking, the edge weight will be increased. Such information is obtained through the Google Maps $\mathrm{API}^{2}$ : for each pair of nodes $\left(e_{i}, e_{j}\right)$ the system asks Google for the traveling time from $e_{i}$ to $e_{j}$ and the traveling time to $e_{j}$ to $e_{i}$, thus creating the edge. Starting and end nodes are slightly different from the other nodes: while the latter have both incoming and outgoing edges, the starting node has only outgoing edges, the end node only incoming edges. Once inferred all the edges, a complete graph from the starting node to the end node is obtained. Then, a routing algorithm is executed on it. Such algorithm is designed based on the one presented in [4]. More specifically, starting from the itinerary comprised by the only starting and end points, further POIs are gradually inserted until all the available time has been spent. Such insertion is not random, but occurs while sorting the left POIs based on several factors, such as popularity and distance. The routing algorithm returns many itineraries from the starting node to the end node. In order to

\footnotetext{
${ }^{1}$ https://foursquare.com.

2 https://developers.google.com/maps/.
} 
obtain the first $k$ of them, which maximize the user's satisfaction, the following scoring function is used:

$$
\text { score }=\alpha \sum_{i=1}^{n} \operatorname{pop}\left(v_{i}\right)-\beta \sum_{i=1}^{n-1} \operatorname{dist}\left(v_{i}, v_{i+1}\right)+\gamma f(n)+\delta \sum_{i=1}^{n} \operatorname{sim}\left(u, v_{i}\right)+\theta \sum_{i=1}^{n} \operatorname{soc}\left(v_{i}\right)
$$

Such function is made up of several terms. All of them are normalized and weighed by constants whose values were set after empirical analysis. The first term denotes the relevance/popularity level of individual POIs. The popularity level of an itinerary is calculated by summing all the users' check-ins in each POI. The second term represents the total distance of the itinerary, which is given by the sum of the traveling times of each single path. This term is the only negative one, in order to give greater relevance to the shorter routes than the longer ones. The third term takes into account the number of venues in the path. The fourth term expresses the path affinity with the user's taste: for every POI, its affinity with respect to the user's interests is assessed. Such value is computed through the cosine-similarity function between the weight vector representing the user profile and weight vector representing the POI category. The last term gives the social contribution, which includes information derived from social networks. The assumption behind this is that if some friends of the active user perform check-ins in a given POI, then such POI receives a bonus value depending on the check-ins amount.

\section{Experimental Evaluation}

This section summarizes the preliminary outcomes of the experimental evaluation. Tests were performed on a sample of 40 real users aged between 20 and 65 years, mostly students and academics. Almost all testers had an active account on one or more social networks. First of all, we created five different scenarios with different contexts, so as to have most possible varied situations. An example is: "suppose yourself to be in Rome, in Termini Railway Station, it is raining, on Monday, at $2 \mathrm{pm}$, by car, traffic is heavy; you have six hours to get to Piazza Navona". Each tester was presented with one of these five scenarios randomly chosen. Based on the scenario and the user profile, the systems returns the first ten itineraries, for each of which the user has to express her satisfaction through a 5-point Likert scale. Itineraries are randomly returned to the target user, so as to preserve the rating fairness. The performance of the recommender was assessed in terms of the normalized version of Discounted Cumulative Gain (nDCG) [5]. $n D C G$ is usually truncated at a particular rank level to emphasize the importance of the first retrieved documents. To focus on the top-ranked items, we considered the $D C G @ n$ by analyzing the ranking of the top $n$ itineraries in the recommended list with $n$ from 1 to 10 . The measure is defined as follows:

$$
n D C G @ n=\frac{D C G @ n}{I D C G @ n}
$$

and the Discounted Cumulative Gain (DCG) is defined as:

$$
D C G @ n=\operatorname{rel}_{1}+\sum_{i=2}^{n} \frac{\mathrm{rel}_{i}}{\log _{2} i}
$$


where rel $_{i}$ is the graded relevance of the $i$-th result (i.e., $0=$ non-significant, $1=$ significant and $2=$ very significant), and the Ideal DCG $(I D C G)$ for a query corresponds to the $D C G$ measure where scores are resorted monotonically decreasing, that is, the maximum possible $D C G$ value over that query. $n D C G$ is often used to evaluate search engine algorithms and other techniques whose goal is to order a subset of items in such a way that highly relevant documents are placed on top of the list, while less important ones are moved lower. Basically, higher values of $n D C G$ mean that the system output gets closer to the ideal ranked output. The graph shown in Fig. 1 illustrates the average values of $n D C G$ for each position, based on the ratings of 40 users. The $x$-axis reports the rank (from 1 to 10), while the $y$-axis displays the respective value of $n D C G$. Analyzing the obtained results, we can notice how the system allowed us to achieve high $n D C G$ values, showing high accuracy performance.

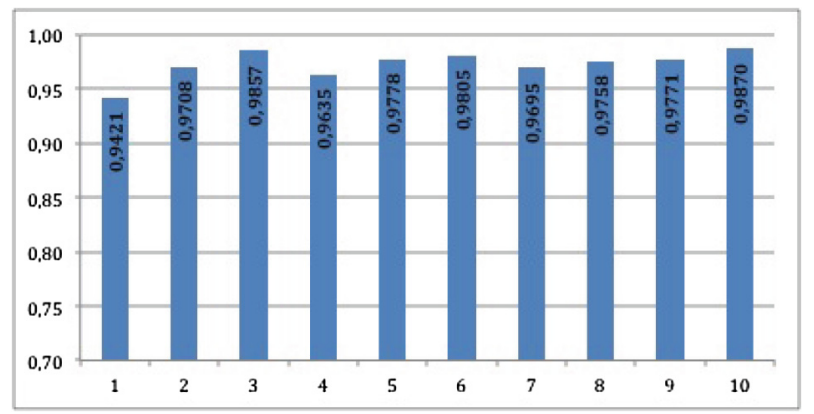

Fig. 1. Values of $n D C G$ for different rank levels.

\section{Related Work}

In the research literature, there are several approaches to making context-aware mobile interaction available in scenarios where users are looking for categories of points of interest (POIs), such as cultural events and restaurants, through remote location-based services [6]. Time constraints are in general more sensitive in itinerary recommendation. Determining the proper visiting time of each place and the proper transit time from one place to another is fundamental for defining route goodness functions [7]. Yoon et al. [8] explicitly model both the available time of the user and the staying time for each POI included in the itinerary. Techniques based on signal processing are proposed for including time dimension in context-aware recommendation tasks $[9,10]$. Online photo sharing services, such as Flickr, or real-world public datasets of rich photographers' histories are often used as sources for mining popular venues [11], travel sequences [12] or, more in general, their attractiveness [13]. The large amount of geo-tagged photos shared on SNS allow LBS to mine also demographic information about the locations 
by detecting people attributes by means of image analysis techniques. In [14] the authors take into consideration several visual features to classify each photo in one of the following attributes: family, friends, couple and solo traveler. By sorting the geo-tagged photos, the followed itineraries are collected and one of the above-mentioned attributes is identified and assigned to each of them. Finally, a Bayesian learning model sorts out the best itinerary given a query and the attributes representing the user profile. Local travel experts can help populate a knowledge base of popular itineraries. Manual customization of the suggested itineraries by the users can provide valuable feedback for improving the local knowledge base [15]. More complex approaches dynamically suggest new POIs according as the last visited ones, their characteristics and categories, personalizing the recommendation as the current context evolves. Multiple conflicting criteria and undesirable situations that may result in the modification of the current schedule can also be considered by monitoring the user's behavior [16].

\section{Conclusions and Future Works}

In this paper, we have described a context-aware, personalized, recommender system of popular itineraries, which takes into account the social contribution as well. Experimental tests showed significant values in terms of $n D C G$, thus indicating that the suggested itineraries were very close to the ones preferred by the target user. Although the results obtained through the proposed recommender are encouraging, there exist several possible future developments of this work. First, we would like to consider more social networks in the data collection, in order to further refine and customize the recommendation process. An other interesting point would be also to relate the influence of the social factor to each user. The idea is to make such weight to increase or decrease based on the user's past behavior. For instance, if in the past the user has liked the POIs visited by her friends (e.g., giving them check-ins), the value of the social factor may be consequently increased by a certain percentage. Finally, we would like to integrate the recommendation process with an analysis of the user-generated content on social networks. Indeed, in the research literature it has been shown that the accuracy of RSs can be improved by considering sentiments and opinions extracted from the user micro-posts [17-19].

\section{References}

1. Biancalana, C., Gasparetti, F., Micarelli, A., Sansonetti, G.: An approach to social recommendation for context-aware mobile services. ACM Trans. Intell. Syst. Technol. 4(1), 10:1-10:31 (2013)

2. Biancalana, C., Gasparetti, F., Micarelli, A., Sansonetti, G.: Social semantic query expansion. ACM Trans. Intell. Syst. Technol. 4(4), 60:1-60:43 (2013)

3. Dey, A.K.: Understanding and using context. Pers. Ubiquitous Comput. 5(1), 4-7 (2001) 
4. Hagen, K., Kramer, R., Hermkes, M., Schumann, B., Mueller, P.: Semantic matching and heuristic search for a dynamic tour guide. In: Frew, A.J. (ed.) Proceedings of the International Conference in Innsbruck, Austria, pp. 149-159. Springer, Vienna (2005)

5. Järvelin, K., Kekäläinen, J.: Cumulated gain-based evaluation of IR techniques. ACM Trans. Inf. Syst. 20(4), 422-446 (2002)

6. Biancalana, C., Flamini, A., Gasparetti, F., Micarelli, A., Millevolte, S., Sansonetti, G.: Enhancing traditional local search recommendations with context-awareness. In: Konstan, J.A., Conejo, R., Marzo, J.L., Oliver, N. (eds.) UMAP 2011. LNCS, vol. 6787, pp. 335-340. Springer, Heidelberg (2011)

7. Hsieh, H.P., Li, C.T., Lin, S.D.: Measuring and recommending time-sensitive routes from location-based data. ACM Trans. Intell. Syst. Technol. 5(3), 45:1-45:27 (2014)

8. Yoon, H., Zheng, Y., Xie, X., Woo, W.: Social itinerary recommendation from user-generated digital trails. Pers. Ubiquitous Comput. 16(5), 469-484 (2011)

9. Biancalana, C., Gasparetti, F., Micarelli, A., Miola, A., Sansonetti, G.: Contextaware movie recommendation based on signal processing and machine learning. In: Proceedings of the 2nd Challenge on Context-Aware Movie Recommendation. CAMRa 2011, pp. 5-10. ACM, New York (2011)

10. Arru, G., Feltoni Gurini, D., Gasparetti, F., Micarelli, A., Sansonetti, G.: Signalbased user recommendation on twitter. In: Proceedings of the 22nd International Conference on World Wide Web, WWW 2013 Companion, pp. 941-944. ACM, New York (2013)

11. Brilhante, I., Macedo, J.A., Nardini, F.M., Perego, R., Renso, C.: Where shall we go today?: planning touristic tours with tripbuilder. In: Proceedings of the 22nd ACM International Conference on Conference on Information \& Knowledge Management, CIKM 2013, pp. 757-762. ACM, New York (2013)

12. Zheng, Y., Xie, X.: Learning travel recommendations from user-generated GPS traces. ACM Trans. Intell. Syst. Technol. 2(1), 2:1-2:29 (2011)

13. Waga, K., Tabarcea, A., Franti, P.: Recommendation of points of interest from user generated data collection. In: International Conference on Collaborative Computing: Networking, Applications and Worksharing, October 2012, pp. 550-555 (2012)

14. Chen, Y.Y., Cheng, A.J., Hsu, W.: Travel recommendation by mining people attributes and travel group types from community-contributed photos. IEEE Trans. Multimedia 15(6), 1283-1295 (2013)

15. Schaller, R., Elsweiler, D.: Itinerary recommenders: how do users customize their routes and what can we learn from them? In: Proceedings of the 5th Information Interaction in Context Symposium, pp. 185-194. ACM, New York (2014)

16. Takayuki, S., Munenobu, N., Naoki, S., Yoshihiro, M., Keiichi, Y., Minoru, I.: A personal navigation system with functions to compose tour schedules based on multiple conflicting criteria. IPSJ Digital Courier 46(11), 2590-2598 (2005)

17. Gurini, D.F., Gasparetti, F., Micarelli, A., Sansonetti, G.: iSCUR: interest and sentiment-based community detection for user recommendation on twitter. In: Dimitrova, V., Kuflik, T., Chin, D., Ricci, F., Dolog, P., Houben, G.-J. (eds.) UMAP 2014. LNCS, vol. 8538, pp. 314-319. Springer, Heidelberg (2014)

18. Feltoni Gurini, D., Gasparetti, F., Micarelli, A., Sansonetti, G.: Enhancing social recommendation with sentiment communities. In: Wang, J., et al. (eds.) WISE 2015. LNCS, vol. 9419, pp. 308-315. Springer, Heidelberg (2015). doi:10.1007/ 978-3-319-26187-4_28

19. Feltoni Gurini, D., Gasparetti, F., Micarelli, A., Sansonetti, G.: Analysis of sentiment communities in online networks. In: SIGIR (2015) 